\title{
Aborto. Análisis constitucional entre el derecho a la vida y la autodeterminación de la mujer
}

\begin{abstract}
Abortion Constitutional analysis between the right to life and self-
\end{abstract} determination of women

1 Natalia Martha Naranjo Ortiz Universidad de Guayaquil, Guayaquil, Ecuador. natalia.naranjoo@ug.edu.ec vida y la autodeterminación de la mujer. AlfaPublicaciones, 4(1.1), 17-29. https://doi.org/10.33262/ap.v4i1.1.137

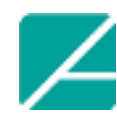

Ciencia Digital

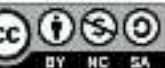

ALFA PUBLICACIONES, es una Revista Multidisciplinar, Trimestral, que se publicará en soporte electrónico tiene como misión contribuir a la formación de profesionales competentes con visión humanística y crítica que sean capaces de exponer sus resultados investigativos y científicos en la misma medida que se promueva mediante su intervención cambios positivos en la sociedad. https://alfapublicaciones.com

La revista es editada por la Editorial Ciencia Digital (Editorial de prestigio registrada en la Cámara Ecuatoriana de Libro con No de Afiliación 663) www.celibro.org.ec

Esta revista está protegida bajo una licencia Creative Commons Attribution Non Commercial No Derivatives 4.0 International. Copia de la licencia: http://creativecommons.org/licenses/by-nc-nd/4.0/ 


\section{Palabras claves:}

aborto, derecho a la

vida,

autodeterminación, empoderamiento.

\section{Keywords:}

abortion, right to

life, self-

determination,

empowerment.

\section{Resumen}

El objetivo general de la investigación es desarrollar un análisis sobre el aborto derivado por estos extremos, desde un punto de vista legal, social y cultural. La metodología utilizada es de tipo documental. Como conclusión, la práctica del aborto es penalizado, no solo para las mujeres sino también para aquellas personas o profesionales que están involucradas en este acto, lo cual conlleva a determinar que Ecuador respeta el derecho a la vida de las personas, desde su gestación; a través de lo que manifiesta la carta magna, como el COIP, donde el aborto no es punible si y solo si la vida de la mujer embarazada corre peligro de muerte o si la misma sufre alguna discapacidad mental; en cambio, los derechos a la salud reproductiva y el derecho a la sexualidad responsable son limitados; en este sentido, se ha manifestado la importancia de la autodeterminación y el empoderamiento de la mujer dentro de todos los espacios sociales, económicos, políticos y culturales, sin tomar en consideración alguna reforma que cambia la percepción de la penalización del aborto, prevaleciendo la cultura conservadora dentro de los organismos competentes

\section{Abstract}

The general objective of the research is to develop an analysis of abortion derived from these extremes, from a legal, social and cultural point of view. The methodology used is documentary type. In conclusion, the practice of abortion is penalized, not only for women but also for those people or professionals who are involved in this act, which leads to determine that Ecuador respects the right to life of people, from their gestation; Through what is stated in the Magna Carta, such as the COIP, where abortion is not punishable if and only if the life of the pregnant woman is in danger of death or if she suffers some mental disability; In contrast, the rights to reproductive health and the right to responsible sexuality are limited; In this sense, the importance of self-determination and empowerment of women has been manifested within all social, economic, political and cultural spaces, without taking into consideration any reform that changes the perception of 
the criminalization of abortion, with the prevailing culture conservative within the competent bodies.

\section{Introducción}

El aborto es un tema que ha generado diversos matices dentro de las sociedades. Ha producido implicaciones éticas, morales, sociales y religiosas, así como ha sido prohibido o limitado en diversas sociedades y permitido en otras, aunque los abortos continúan siendo comunes incluso donde la presión social o la ley se oponen a ellos (Doblado-Donis et al., 2010).

Sin embargo, la complejidad de este tema tiene otro extremo, que lo ve desde un punto de vista filosófico, donde el derecho a la vida radica en aquellas personas independientes y con razonamiento lógico. Esta comunidad manifiesta que la fecundación es genotípicamente humano, aunque fenotípicamente no se puede hacer una aseveración tan radical, por lo que el ser humano existe cuando nace, "está ahí" en el mundo externo (Triana-Agudelo et al., 2012). Esto es lo que da el origen a la vinculación de la autodeterminación de la mujer en decidir sobre su propio cuerpo.

Una de las exigencias que se resaltan por parte de los sectores feministas es la voluntad de que las mujeres puedan decidir sobre su cuerpo. Quieren que las sociedades las contemplen como forjadoras de sus propios destinos, librándose del estigma de que son las matriarcas de hogares. Quieren decidir sobre la responsabilidad de ser madres cuando no han podio lograr sus propias metas y planes de vida. En otras palabras, promueve nuevas conceptualizaciones, entre las que se encuentra la de la interrupción voluntaria del embarazo como un derecho de las mujeres, un derecho a la autodeterminación reproductiva, vinculado al principio más amplio de autonomía corporal que es el derecho a la integridad física (Lamas, 2008).

Por lo tanto, es importante poder desarrollar un análisis sobre el aborto derivado por estos extremos, como el derecho a la vida y la autodeterminación de la mujer sobre decidir sobre su cuerpo, desde un punto de vista legal, social y cultural. La metodología utilizada es de tipo documental que proponga los conceptos que permitan el desarrollo del objetivo general de la presente investigación.

Es necesario poder definir el aborto con el objetivo de poder darle una figura social, política y cultural a este fenómeno; además de poder configurarle aspectos jurídicos que puedan otorgarle peso de ley en situaciones que perjudiquen la calidad de vida de las mujeres y del no nato. Según Astete et al. (2014), define el aborto de la siguiente manera: 
Después de la viabilidad, la interrupción del embarazo se considera inducción de parto prematuro. Si la intención es la eliminación del feto se considera aborto tardío (late-term abortion). Aborto en sentido jurídico es toda maniobra destinada a interrumpir el embarazo impidiendo que llegue al término natural, con destrucción o muerte del producto. (p. 1450)

No obstante, desde el punto de vista regulatorio lo que más incide es cómo se interrumpe el embarazo, no cuando. Esto tiene que ver con el efecto de realizar abortos en sociedades que penalizan dicha situación y en la cual se realizan de manera clandestina corriendo peligro la vida de la mujer. Todo esto es lo que desencadena en la fragilidad de la mujer ante el acto de aborto. Esta situación influenciada por la desinformación, los riesgos, el miedo a exponer el propio cuerpo, la soledad, la circulación de imágenes desoladoras, lo cual produce una matriz que busca mantener el aborto en las sombras, centrada en el estigma y en el tabú social que pesan sobre la práctica (Szwarc \& Vázquez, 2018).

Ante estas situaciones prevalece dos factores extremos el cual involucra el derecho a la vida y la autodeterminación de la mujer a decidir sobre su cuerpo. Por tal motivo, es necesario contraponer las características, importancia y la implicación de estas dentro del marco legal de Ecuador. Por esta razón, el derecho a la vida es un factor de importancia a la hora de establecer las pautas a considerar legalmente en el aborto. El concepto de vida viene epistemológicamente del latín vita asociado con la raíz bios, y a la palabra zoon que significa animal y ser vivo (Borbón et al., 2020).

Del mismo modo según Erazo-Bustamante (2017) señala que la vida es un derecho fundamental, consagrado en las Cartas Magnas de los diferentes países, se trata de un derecho que precede a los restantes derechos, debido a que, si desaparece el titular del derecho a la vida, desaparece cualquier otro derecho posible.

Pero ¿desde cuándo comienza el derecho a la vida?, este es el tema que resulta de interés dentro de las diferentes aristas de la sociedad. Esta situación ha dado generado una serie de pugnas por el derecho a las mujeres de ser autodeterminadas en función de decidir sobre su cuerpo a través de los mecanismos del aborto. En este sentido, es necesario comprender donde comienza la vida de una persona. Según el fundamento jurídico 5 de España, citado por Gómez-Montoro (2018), señala que "la vida humana es un devenir, un proceso que comienza con la gestación (...), y que termina con la muerte, así como que la gestación genera un tertium existencialmente distinto de la madre” (p. 52).

No obstante, la disyuntiva radica es que para algunos sectores de la sociedad el derecho a la vida no radica en el momento de la gestación sino al momento de nacer. Aunque existen políticas que manifiestan lo contrario, las cuales son basados en el ámbito jurídico, donde se considera el derecho a la existencia desde el mismo momento de que el ser humano está en estado embrionario. Esta situación ha mantenido a Ecuador dentro de los 
países que garantizan la vida. Esto ha impulsado a ratificar acuerdos internacionales que buscan la protección de la vida; y, facilitar el resarcimiento de daños frente a la vulneración de derechos producidos al interno de un país (Cueva-Flores \& Eguiguren, 2019).

Por otra parte, el empoderamiento de las mujeres ha estado en la palestra en los últimos años, donde las sociedades exigen mayor protagonismo de las mujeres en las labores civiles de toda índole. A medida del crecimiento de la globalización, también a crecido el deseo del género femenino en retomar espacios que nunca han sido valoradas para ello. Unos de los aspectos que se consideran en el empoderamiento femenino es el derecho a la reproducción, derecho a utilizar métodos anticonceptivos efectivos y derecho a decidir sobre su cuerpo. Cada una de estas aristas permite generar la libertad y la decisión sobre el futuro de las mujeres y de cómo quieren desarrollar la vida.

Asimismo, han existido mecanismo en defensa y comunicación de estos tipos de empoderamientos a través de congresos, simposio o cumbres. Tal es el caso de la cumbre de Nairobi realizada en el 2019, donde se realizaron debates sobre el derecho a la garantía e integridad corporales. Por tal motivo, se han fijado tres objetivos que permitirán eliminar parte de la vulnerabilidad de las mujeres. Para el 2030 se plantea lograr 3 ceros, cero mortalidades maternas, cero necesidades insatisfechas de anticonceptivos y cero violencias sexuales, de género y prácticas nocivas; por lo cual, está implícito el pleno ejercicio de la autonomía corporal por parte de todas las mujeres y niñas (Erken, 2021).

\section{Metodología}

La investigación documental se concreta exclusivamente en la recopilación de información de diversas fuentes, con el objeto de organizarla describirla e interpretarla de acuerdo con ciertos procedimientos que garanticen confiabilidad y objetividad en la presentación de los resultados (Palella \& Martins, 2010). Para lograr este propósito se utilizó herramientas como fuentes primarias. Según Hernández-Sampieri et al. (2014), señalan que las fuentes primarias más utilizadas son libros, artículos de revistas científicas y ponencias o trabajos presentados en congresos, simposios y eventos similares, debido a que son las que sistematizan la información, profundizan más en el tema que desarrollan y son altamente especializadas.

\section{Resultados y Discusión}

\section{El aborto en Ecuador}

Uno de los temas más importante durante los últimos años en las diferentes sociedades es el tema del aborto, porque involucra aspectos sociales, económicos, culturales, religiosos, entre otros. Estos mismos debates que suceden en el mundo se han realizado en Ecuador. En la asamblea constituyente para el desarrollo de la carta magna del 2008, se han 
realizado debates para implementar políticas y mecanismos que involucran el empoderamiento de la mujer.

Del mismo modo, uno de los mecanismos creados como es el Código Orgánico Integral Penal (COIP) realizó unas reformas en el año 2014, donde incrementó la penalización por aborto en mujeres. Esto precipitó una serie significativa de enjuiciamiento a mujeres que han realizado el aborto. Antes de este debate las denuncias por aborto eran casi inexistentes y no había un registro de estas (Zaragocin et al., 2018). Después de la modificación del COIP se establece la ilegalidad del aborto inducido consentido, con penas privativas de la libertad tanto para la persona que haga abortar a una mujer (uno a tres años) y la mujer que lo permita (seis meses a dos años) (Imbago \& Meneses, 2018).

Esto último resalta la importancia de la penalización a las personas que ejecutan el aborto, colocando en peligro de vida a las mujeres, debido a que en muchas ocasiones son realizadas de manera clandestina, ante la espalda de los ojos de la ley. Esta situación, expone a la mujer, ya que a veces no tiene conocimiento de lo que le realizarán, por motivo de que está inconsciente, no posee la suficiente capacidad para comprender las cosas o aparecen otras personas toman decisiones sobre su cuerpo sin su consentimiento (Granizo-Bustamante \& Veloz-Cherrez, 2019).

Asimismo, dentro de la reforma del COIP existen dos causas que permiten que no sea punible el aborto: si está en peligro la vida de la mujer y si es producto de una violación a una mujer con discapacidad mental. Lo que demuestra es que la principal ley sigue estando a favor de la penalización del aborto, sin considerar las opiniones de las mujeres y a su autodeterminación, prevaleciendo el derecho a la vida, desde el momento de la gestación. No obstante, para el año 2019 entró en discusión la despenalización del aborto en la Asamblea Nacional, Esta situación no logro cumplir con los objetivos de poder salvaguardar la integridad de las mujeres en los casos de realizar aborto por causas como violación, incesto, estupro e inseminación no consentida (Pillalaza-Piguave, 2020).

Derecho a la vida versus la autodeterminación de la mujer en decidir sobre su cuerpo en Ecuador

El tema del aborto es muy complejo debido a que toca las fibras más sensibles de la sociedad. La misma constitución de la República de Ecuador muestra contrariedad debido a que señala en los artículos 43 al 45 tema que reflejan el derecho a la vida desde la concepción, pero en el 63 muestra el respecto a la autodeterminación parcial de la mujer a través del derecho a la salud y reproducción, otorgando una puerta abierta para el derecho al aborto. Los artículos 45 y 63 de la carta magna expresan consideraciones, que demuestran la luz que pudiera existir en la despenalización del aborto. 
En el artículo 45 de la constitución de la república determina que el Estado reconocerá y garantizará la vida, incluido el cuidado y protección desde la concepción, por lo que deja por sentado que el Código Penal no es el único que protege al producto de la concepción (León-González, 2020). En el artículo 63 refleja que, al dotar de autonomía a los derechos reproductivos, se busca que las decisiones sobre reproducción se produzcan sin interferencias en un marco de respeto sobre si tener o no hijas o hijos, así como el número e intervalo de los mismos (Espinoza-Plúa, 2018). De la misma forma, esta ambigüedad ha permitido prevalecer la penalización del aborto, lo cual ha incidido en que las mujeres opten por decisiones desesperadas, insalubres y riesgosas, que en muchas ocasiones terminan con su vida, por lo cual la norma no está cumpliendo con su fin (López et al., 2021).

En definitiva, la jurisprudencia defiende la vida del nasciturus, al igual que prevalecen los derechos a las mujeres de tener derechos sexuales y reproductivos. Es así como, es necesario desarrollar los derechos del nasciturus tal como lo concibe Bustamante et al. (2019), donde lo representa como un ser humano, por lo que debe admitirse que constitucionalmente tiene derecho a la vida, sea considerado o no por la legislación civil como persona. El respeto al ser humano desde la concepción también radica en el respeto de la madre en la etapa de gestación. En el numeral 3 del artículo 43 de la constitución se velan por los derechos de las mujeres en su salud integral desde el embarazo, parto y postparto (Zambrano-Figueroa \& Barreiro-Loor, 2019).

Sin duda alguna, el derecho a la vida es un derecho universal que esta descrito en todos los tratados internacionales y en todas las cartas magnas de los países. En este sentido, la defensa del nascirutus es una prioridad para la legislación ecuatoriana.

El derecho a la vida es un derecho natural; en otras palabras, es un principio jurídico que genera la propia naturaleza, es aquella que debe ser tomada en cuenta para la elaboración e interpretación de una norma que se va a establecer en el derecho positivo, que se refiere a las normas ya escritas, a las normas emanadas por el poder soberano del Estado, el cual se aplicará a los casos que se presenten en la sociedad. (Coronel-Ortiz, 2019, p. 45)

A razón de esta situación, el Estado ecuatoriano debe proteger los derechos a todo aquel que tiene vida, por lo que debe crear los mecanismos para fomentar tal derecho, en todos los aspectos legales del estado civil y jurídico. Para muchos ciudadanos las leyes deben impedir el atentado contra la vida, en especial la del nascirutus, por lo que estos derechos son principios jurídicos dentro de Estado. La jurisprudencia debe velar, de la misma forma, en la abstención de cualquier daño que se le pueda hacer a cualquier forma de vida, esto quiere decir que aquellas personas involucradas en el acto de poner en peligro la vida, también deben ser penalizados, porque el desconocimiento de la ley no lo inhibe de sus responsabilidades. 
De la misma forma, la penalización sobre violentar la vida del nascirutusse detalla dentro de los artículos del COIP de Ecuador, específicamente en el Capítulo Segundo que trata sobre Delitos contra los Derechos de Libertad, dentro de la Sección Primera que manifiesta los Delitos contra la Inviolabilidad de la Vida. Estos artículos son: 147, aborto con muerte; 148, aborto no consentido; 149, aborto consentido; y, 150, aborto no punible. Todo lo anterior, conlleva nuevamente a la realización acelerada de abortos clandestinos donde peligra la vida de la mujer embarazada.

Para evitar tales situaciones, se han planteado mecanismos que pueden evitar la prevalencia de abortos clandestinos, tal como es el caso de los partos anónimos. Esta situación permite, también, el abandono de niños, lo cual es penado por el estado ecuatoriano. Por lo cual, es un mecanismo jurídico alternativo, que se ubica por detrás de la penalización absoluta del aborto y por delante de la legalización de este, como recurso plausible entre los peores males posibles que atentan contra la vida del nasciturus y contra la estabilidad de la madre (Cueva-Flores \& Eguiguren, 2019).

Por otra parte, se coloca sobre el otro extremo de la balanza los derechos sexuales y reproductivos de las mujeres a través de la autodeterminación que buscan para decidir sobre su cuerpo. Esto significa que las mujeres son las que deciden como y cuando ser madres, por lo que ya no es una carga llevar la batuta del estándar de la familia, lo que conlleva que para ellas el derecho al aborto es una de las prioridades. Iñiguez-Manso \& Peredo-Rojas (2017), comparten esta situación de la siguiente manera:

El término "derechos sexuales y reproductivos" se ha desarrollado al alero de la ideología de género, corriente que considera a la maternidad como una forma de opresión contra la mujer. Los derechos sexuales y reproductivos implican, por lo tanto, el derecho al "ejercicio sin límites de la sexualidad apetecida por cada uno, sin contraer ninguna enfermedad" 43. La frase "sin ninguna limitación" significa que la mujer es la que decide, a voluntad, los mecanismos por medio de los cuales evita el embarazo o sus consecuencias. El aborto se convierte en un "derecho" que no respeta ningún otro, ni si quiera la libertad de conciencia de los facultativos médicos. (p. 47)

Asimismo, el Estado debe promover mecanismos que puedan garantizar los derechos de las mujeres más allá de los derechos de vida, sino también sean las propietarias de su situación sexual y reproductiva. Las leyes emanadas de los organismos públicos deben buscar, no solo establecer las normativas que garanticen la autodeterminación, sino que deben ir más allá de unas normas legales, sino que ayuden a evitar trabas en el cumplimiento de la ley. Esta situación disminuye las decisiones de las mujeres a realizar abortos clandestinos. Los anterior se describe como respuesta de que obligan a las mujeres a continuar con embarazos que no son viables en contra de su voluntad, lo que genera una violación a una serie de derechos humanos reconocidos (Erken, 2021). 
Ecuador no escapa de esta realidad, varias organizaciones de mujeres a nivel local y nacional han denunciado los obstáculos que experimentan las mujeres cuando demandan del sistema judicial la protección de sus derechos, así como las omisiones de la política pública ejecutada por el Gobierno de la Revolución Ciudadana, paradójicamente, promotor de la nueva Constitución (Mendoza-Eskola, 2018). Otros factores que inciden en la desigualdad de género es que siempre el modelo es el hombre, el cual puede decidir sin prejuicios y con el apoyo de la jurisprudencia. Esto lo corrobora Mendoza-Eskola (2019) donde el derecho únicamente norma el cuerpo femenino, por lo que las mujeres son presentadas como "el otro" del sujeto que sirve de modelo.

Es así como la jurisprudencia, para este sector, debe cambiar la percepción de cómo ve a la mujer y la importancia que tiene sobre la sociedad. Debe velar más allá del derecho a la vida, sino también sus derechos a ser auto determinante sobre el destino de la misma vida que tiene como garantía. En este sentido, esta situación pudiera quedar reducida que en el caso de la violación queda a salvo porque la agresión que sufre la mujer se "subsana" con la muerte del embrión (Iñiguez-Manso \& Peredo-Rojas, 2017).

Esta misma situación ha permitido que, dentro del seño de las mujeres nazca lo que se denomina el empoderamiento. El empoderamiento se caracteriza por: empoderarse de manera personal se concreta en la individuación, es decir, en la transformación personal en un ser individual: único e independiente, con personalidad y concepciones propias, con capacidad de decidir y de actuar por cuenta propia, con movilidad y autodeterminación. La autoestima, la seguridad y la confianza se incrementan al empoderarse. Y cuando empoderarse se produce en grupos y movimientos, se condensa en la conciencia de tener una identidad grupal específica.

\section{Conclusiones}

- El aborto se ha convertido en un tema controversial por lo significativo en el derecho a la vida, así como aun punto clave en la autodeterminación de la mujer para decidir sobre su cuerpo. En la gran mayoría de los países en vías de desarrollo, la práctica del aborto es penalizado, no solo para las mujeres sino también para aquellas personas o profesionales que están en este acto. Esto mismo sucede en Ecuador donde la constitución de la república, como el COIP penalizan por privativa de libertad a las mujeres que practiquen estos procedimientos.

- Esta situación conlleva a determinar que Ecuador respeta el derecho a la vida de las personas, desde su gestación. La carta magna manifiesta el derecho a la vida del nascirutus, donde el COIP respalda esa decisión manifestando que el aborto no es punible si y solo si la vida de la mujer embarazada correo peligro de muerte o si la misma sufre alguna discapacidad mental. En los últimos se han intentado realizar reformas a las leyes cuando el aborto se quiere realizar por causas como violación, incesto, estupro o inseminación no consentida por la mujer, la cual no 
ha arrojado respuestas satisfactorias al sector que exige sus derechos de escoger y determinar su propio futuro. siempre domina ante los ojos del hombre y de dios el derecho a la vida sin importar las consecuencias que puedan traer para todos los involucrados.

- Es aquí donde los derechos a la salud reproductiva y el derecho a la sexualidad responsable son limitados. La constitución de la república ecuatoriana ha manifestado la valoración a estos derechos dentro de sus artículos, manifestando la importancia de la autodeterminación y el empoderamiento de la mujer dentro de todos los espacios sociales, económicos, políticos y culturales. Sin embargo, no involucra alguna reforma que cambia la percepción de la penalización del aborto, prevaleciendo la cultura conservadora dentro de los organismos competentes. Los cambios a favor del empoderamiento de las mujeres no se han producido como tal, debido a que muchos organismos que materializan los mandates jurídicos no realizan los verdaderos cambios a favor de las mujeres. todavía queda camino por recorrer en conseguir un empoderamiento efectivo en la sociedad ecuatoriana, donde la bandera de la despenalización del aborto es la principal en todos los movimientos femeninos.

\section{Referencias Bibliográficas}

Astete, C., Beca, J. P., \& Lecaros, A. (2014). Propuesta de un glosario para la discusión del aborto. Revista médica de Chile, 142(11), 1449-1451.

Borbón, L. D., Fúquene, J. A., \& Solaque, L. X. (2020). Derecho a la vida. Introducción a Derecho. Universidad Católica de Colombia.

Bustamante, S. E., Ochoa, M. E., \& Cruz, H. B. (2019). Respuestas del Estado ecuatoriano frente a la problemática del aborto. Dilemas contemporáneos: Educación, Política y Valores, 1-29.

Coronel-Ortiz, V. (2019). La vida y el derecho civil ecuatoriano. Killkana sociales: Revista de Investigación Científica, 3(3), 39-46.

Cueva-Flores, J. L., \& Eguiguren, C. A. (2019). El derecho a la vida ya la salud de los neonatos abandonados en Ecuador. Ius Humani. Law Journal, 8, 143-168.

Doblado-Donis, N. I., De la Rosa-Batista, I., \& Junco-Manrique, A. (2010). Aborto en la adolescencia un problema de salud. Revista Cubana de Obstetricia y Ginecología; 36(3), 409-421.

Erazo-Bustamante, S. E. (2017). La vida como derecho fundamental de las personas. Ámbito Jurídico. 
Erken, A. (2021). Mi cuerpo me pertenece. Reclamar el derecho a la autonomía y a la autodeterminación. UNEFPA.

Espinoza-Plúa, M. G. (2018). El espejismo laico del Ecuador. Los debates constituyentes sobre el aborto, la adopción homosexual y el nombre de Dios en el preámbulo de la Constitución. Revista de Derecho, No. 29, 135-168.

Gómez-Montoro, Á. J. (2018). El Estatuto Constitucional del no nacido: evolución y situación actual en España. Revista de Derecho Político, 1(102), 47-78.

Granizo-Bustamante, D. D., \& Veloz-Cherrez, J. C. (2019). Impacto del aborto en Ecuador en adolescentes víctimas de violaciones según el Código Orgánico Integral Penal. Guayaquil, Ecuador: Trabajo de grado para optar al título de Abogado de los Tribunales Juzgados de la República de Ecuador de la Universidad de Guayaquil.

Hernández-Sampieri, R., Fernández-Collado, C., \& Batista, M. d. (2014). Metodología de la investigación, 6ta edición. México, D. F.: McGraw-Hill / Interamericana Editores, S.A. DE C.V.

Imbago Jácome, M. D., \& Meneses Valle, D. E. (2018). Conocimiento y actitudes sobre las leyes del aborto y de las guías de práctica clínica del aborto en Ecuador entre los estudiantes de sexto a décimo segundo nivel de pregrado de la Facultad de Medicina y Posgradistas de Ginecología y Obstetricia de primer. Quito, Ecuador: Trabajo de grado para optar al título de Medico Cirujano de la Pontificia Universidad Católica del Ecuador.

Iñiguez-Manso, A. R., \& Peredo-Rojas, M. I. (2017). Propuestas de solución para los aparentes conflictos jurídicos que se pueden suscitar entre el derecho a la vida del que está por nacer y los derechos de la madre. Revista Derecho Público Iberoamericano, (11), 35-65.

Lamas, M. (2008). El aborto en la agenda del desarrollo en América Latina. Perfiles latinoamericanos, 16(31), 65-93.

León-González, P. A. (2020). Aborto: Una visión general de por qué no es óptimo para el sistema jurídico ecuatoriano permitir el aborto por violación. UDA Law Review, (2), 51-61.

López, M. M., Andreeva, Y. J., \& Velasco, J. P. (2021). Aborto en el Ecuador: Análisis de la sentencia No. 34-19-IN/21. Sociedad \& Tecnología, 4(S2), 529-545.

Mendoza-Eskola, C. (2018). Género y Derecho: Análisis de la jurisprudencia ecuatoriana en torno a los derechos de las mujeres durante el período 1998-2018. In VI 
Encuentro Latinoamericano de Metodología de las Ciencias Sociales 7 al 9 de noviembre de 2018.

Mendoza-Eskola, J. C. (2019). Identidades femeninas en el derecho ecuatoriano. Quito, Ecuador: Derecho y Sociedad.

Palella, S., \& Martins, F. (2010). Metodología de la investigación cuantitativa. Caracas, Venezuela: FEDUPEL, Fondo Editorial de la Universidad Pedagógica Experimental Libertador.

Pillalaza-Piguave, C. A. (2020). La despenalización del aborto en Ecuador 2019: cobertura y fuentes periodísticas. Quito, Ecuador--: Trabajo de grado para optar al título de Licenciado en Periodismo Multimedios de la Universidad San Francisco de Quito.

Szwarc, L., \& Vázquez, S. S. (2018). Lo quería hacer rápido, lo quería hacer ya: tiempos e intervalos durante el proceso de aborto. Sexualidad, Salud y Sociedad (Rio de Janeiro), No 28, 90-115.

Triana-Agudelo, A. M., Soto-Méndez, C., \& Peña-Ocampo, J. J. (2012). El derecho a la vida de los no nacidos. MAGISTRO, 6(11), 87-99.

Zambrano-Figueroa, M. P., \& Barreiro-Loor, T. M. (2019). El aborto en la legislación penal ecuatoriana. Guayaquil, Ecuador: Trabajo de grado para optar al título de Abogado de los Tribunales y Juzgados de la República de Ecuador de la Universidad de Guayaquil.

Zaragocin, S., Cevallos, M. R., Falanga, G., Arraola, I., Ruales, G., Vera, V., \& Yépez, A. (2018). Mapeando la criminalización del aborto en el Ecuador. Revista de bioética y derecho, (43), 109-125.

\section{【L Ciencia}


El artículo que se publica es de exclusiva responsabilidad de los autores y no necesariamente reflejan el pensamiento de la Revista Alfa Publicaciones.

\section{Ciencia}

El artículo queda en propiedad de la revista y, por tanto, su publicación parcial y/o total en otro medio tiene que ser autorizado por el director de la Revista Alfa Publicaciones.
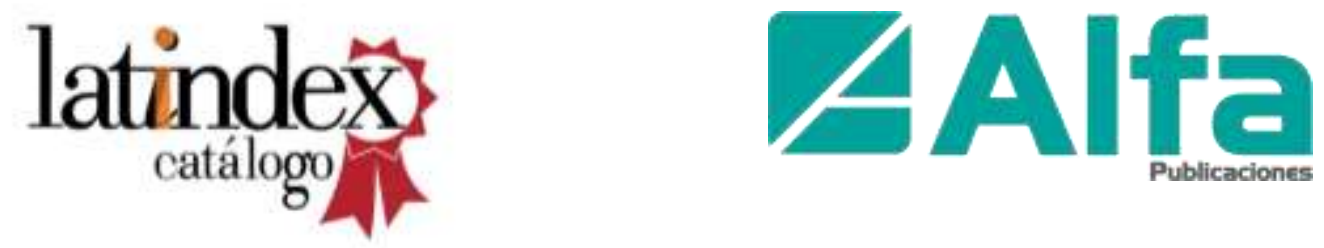

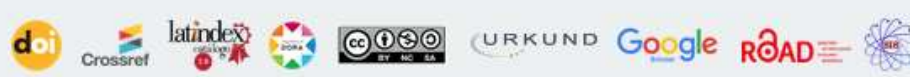
DLatinREV

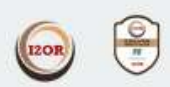

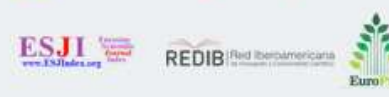
$\underbrace{}_{\text {wizdom.ai }} \bigodot_{\text {OpenAlRE }}^{\oplus}$

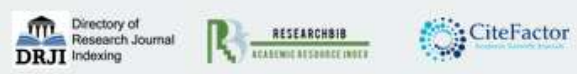

